\title{
A HYBRID OPTIMIZATION METHOD FOR VEHICLE ROUTING PROBLEM USING ARTIFICIAL BEE COLONY AND GENETIC ALGORITHM
}

\author{
M. Davoodi ${ }^{1, *}$, M. Malekpour Golsefidi ${ }^{2}$, M. S. Mesgari ${ }^{3}$ \\ ${ }^{1}$ M.Sc. in GIS at Faculty of Geodesy and Geomatics, K.N.Toosi University of Technology, Iran - Mojtaba.Davoodi@ut.ac.ir \\ ${ }^{2}$ School of Surveying and Geospatial Engineering, College of Engineering, University of Tehran, Iran - Majid.Golsefidi@ut.ac.ir \\ ${ }^{3}$ Faculty of Geodesy and Geomatics, K.N.Toosi University of Technology, Tehran, Iran - Mesgari@kntu.ac.ir
}

Commission VI, WG VI/4

KEY WORDS: GIS, Vehicle Routing Problem, Optimization, Genetic Algorithm, Artificial Bee Colony

\begin{abstract}
:
Vehicle Routing Problem is one of the classic problems in GIS (Geospatial Information System) which had been studied for long times. An answer can be accepted as a good solution if it would be able to optimize the total length of the route or decrease the number of vehicles. A VRP defines finding the optimum route for some vehicles that serve to some customers and return to the service center. This problem is economically important because the cost and the time of serving to costumers are related to optimization of the problem's answer. Furthermore, there are many problems like BUS management, Post pickup and delivery system and other servicing systems, which are technically similar to VRP. The aim of these problems is finding a composition of optimum routes between server and costumers. In addition, as the cost is related to time, finding shortest path means decreasing cost serving and decreasing time. In this article, a hybrid model using Artificial Bee Colony and Genetic Algorithm is proposed to solve VRP. In the first step, Artificial Bee Colony has been used to find a solution for five vehicles. The scout and the onlooker bees produced in 8 modes by two methods including the nearest neighborhood and the wide neighborhood. In the second step, the Genetic Algorithm helps to optimize the solutions. The results show that the production of the scout bees is the most effective factor in the answers to the problem and helps greatly converging the answers as soon as possible.
\end{abstract}

\section{WHAT IS VRP?}

The vehicle routing problem is one of the most important problems in distribution and logistics since the 1960s (Gökçe, E.İ., 2004). It is described as finding the minimum distance of the routes of several vehicles that must serve some customers. This system is described as a weighted graph $\mathrm{G}=(\mathrm{V}, \mathrm{A}, \mathrm{d})$ where the vertices are $\mathrm{V}=\left\{\mathrm{V}_{0}, \mathrm{~V}_{1}, \ldots, \mathrm{V}_{\mathrm{n}}\right\}$, and the arcs are $A=\left\{\left(v_{i}, v_{j}\right): i \neq j\right\}$. A central point where each vehicle starts its route is located at $\mathrm{V}_{0}$ and each vertex represents the customers. The distances associated with each arc are the variabled_ij, which are measured by Euclidean computations. The problem is solved under the following constraints:

1. Each customer is visited only once by a vehicle.

2. Each vehicle must start and end its route at $\mathrm{V}_{0}$.

\section{LITERATURE REVIEW}

Marinakis did a comparative analysis and a survey on Particle Swarm Optimization for the Vehicle Routing Problem. They present a survey paper was a review and brief analysis of various investigations about VRP problem using PSO (Marinakis et al., 2017).

Brandão introduced Metaheuristic for the Vehicle Routing Problem with Backhauls and Time Windows. This article contains two disjoint sets of customers: those that receive goods from the depot, who are called linehaul, and those that send goods to the depot, named backhauls. Each customer is associated with an interval of time (time window), during which each one should be served. For solving this problem, a deterministic iterated local search algorithm was created, which was tested using a large set of benchmark problems taken from the literature. These computational tests have proven that this algorithm competes with the best-known algorithms in terms of the quality of the solutions and computing time (Brandão, 2018).

Marinakis investigated a multi-adaptive Particle Swarm Optimization for the Vehicle Routing Problem with time windows. This paper proposed a new variant of the Particle Swarm Optimization (PSO) algorithm for the solution of the Vehicle Routing Problem with Time Windows (VRPTW). Three different adaptive strategies are used in the proposed Multi-Adaptive Particle Swarm Optimization (MAPSO) algorithm. The first adaptive strategy concerns the use of a Greedy Randomized Adaptive Search Procedure (GRASP) that is applied when the initial solutions are produced, and a new solution is created during the iterations of the algorithm. The second adaptive strategy concerns the adaptiveness in the movement of the particles from one solution to another, where a new adaptive strategy, the Adaptive Combinatorial Neighbourhood Topology, is used. Finally, there is adaptiveness in all parameters of the Particle Swarm Optimization algorithm. The algorithm was compared with other versions of PSO and with the best-performing algorithms from the literature (Marinakis et al., 2019).

\footnotetext{
* Corresponding author
} 


\section{ARTIFICIAL BEE COLONY}

The artificial bee colony algorithm $(\mathrm{ABC})$ is one of the most useful optimization algorithms based on swarm intelligence and Bee's intelligent behavior which originated in 2005. This algorithm resembles the style of eating attitudes of bees. In the initial variant of $\mathrm{ABC}$, the algorithm implements a species of local search that is merged with a random search and can be utilized to optimize the combination of functional optimization. Theodore Vass had proposed using the swarm intelligence of bees to develop synthetic systems aimed at solving complicated enigmas in traffic and transportation (Pham et al., 2006).

Bee's search behavior simulates the Bee's algorithm. It is known for its high stability and functionality as a great optimization tool. Bees randomly seek around flower sites to discover food in the environment. As the process of exploration goes forward, flower sites look better in quality, and other remaining bees are randomly searching to find new fields (Purves et al,. 2003; Najibi and Arabsheibani, 2013). The kinds of bees in ABC are:

- The Employed Bee: It stays on a food source and provides the neighbourhood of the source in its memory (Karaboga, 2005).

- The Follower Bee: It gets the information of food sources from the employed bees in the hive and selects one of the food sources to gather the nectar (Kumar et al., 2014).

- The Scout: It is responsible for finding new food, the new nectar sources (Seeley et al., 1988).

The overall steps of the $\mathrm{ABC}$ are:

- Create an initial population of (n) scout bees with random search and assess the fitness function

- $\quad$ Picked best $(\mathrm{m})$ sites for neighbourhood search: the best e sites and (m-e) other chosen sites

- Define the size of the neighbourhood (patch size)

- Recruit bees for selected sites (more bees for the best $\mathrm{e}=2$ sites)

- Choose the fittest bee from each site

- $\quad$ Specify the (n-m) waiting bees to random search

- Discover the global fittest answer

Movement of the Follower:

Probability of selecting a nectar source is (Tsai et al., 2016):

$$
P_{i}=\frac{F\left(\theta_{i}\right)}{\sum_{k=1}^{S} F_{i}\left(\theta_{i k}\right)}
$$

Where $P_{\mathbb{1}}$ is the probability of selecting the $\mathrm{i}^{\text {th }}$ employed bee, $\mathrm{S}$ is the number of employed bees, $\theta_{i}$ is the position of the $i^{\text {th }}$ employed bee and $F\left(\theta_{i}\right)$ is the fitness value.

Calculation of the new position [10]:

$$
\theta_{i j}(\mathrm{t}+1)=\theta_{i j}(\mathrm{t})+\phi\left(\theta_{i j}(\mathrm{t})-\theta_{k j}(\mathrm{t})\right)
$$

Where $\theta_{i}$ is the position of the follower bee, $t$ is the iteration number, $\theta_{k}$ is the randomly chosen employed bee, $\mathrm{j}$ is the dimension of the solution and $\Phi(\ldots)$ is A series of random variable in the range $[-1,1]$.

The movement of the scout bees follows equation [10]:

$$
\theta_{i f}=\theta_{j \min }+r \cdot\left(\theta_{j \max }-\theta_{j \min }\right)
$$

Where $\mathrm{r}$ is $\mathrm{A}$ random number and $\mathrm{r} €[0,1]$.

\section{SOLVING VRP USING ABC ALGORITHM}

In this part, the structure of the $\mathrm{ABC}$ algorithm sets to solve the VRP problem.

\subsection{The Scout Bees}

An answer is considered to be a string of integers, each number assigned to a particular point. In this paper, 20 points are considered as the customers and one point as the service centre. Meanwhile, 5 vehicles with 4 capacities are included for this issue. The path of each vehicle starts at point 21 (the origin point or service centre) and travels through four points and returns to the starting point. Therefore, the general answer is the set of answers to these 5 services, which is formed as a 26 string numbers and shows below:

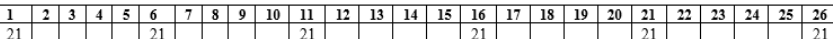

Figure 1. The shape of each answer in algorithm

The method of producing an answer or a scout bee is possible in two ways:

4.1.1 Random method: In this case, a number representing a customer point is randomly selected and awarded by the first vehicle. Then, from the rest of the remaining points, another point is selected randomly and placed in the next position. This will continue as a complete arrangement (an answer) will be created. It should be noted that each point is assigned only to one device, and the capacity of each device is specific.

4.1.2 Nearest neighbourhood method: In this method, for the first vehicle, a point is chosen randomly and assigned to it. Then, according to the distance between the points, the 3 remaining options (points or customers) are selected from the closest points to the first selected customer. Furthermore, this trend will be applied to other vehicles, too. It should be noted that no repetitious point will be selected in vehicles.

\subsection{The Onlooker Bees}

The onlooker bee is trying to improve this answer by changing the answer that is related to scout bee. For each scout bee, 4 onlooker bees are produced. These four answers are made by using two strategies: close neighborhood and far neighborhood.

4.2.1 Far Neighbourhood: These neighbourhoods are created with great variations in existing answers.

4 Onlooker are produced as follows:

Onlooker 1: One point from each route of a vehicle will be given to the next vehicle's route

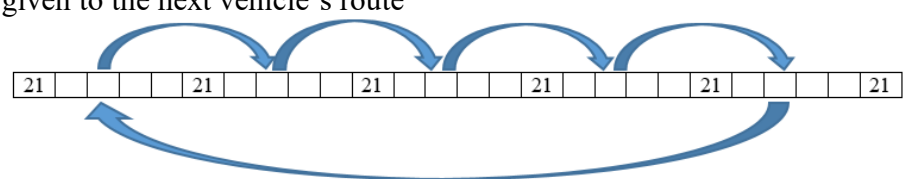

Figure 2. The format of Onlooker 1

Onlooker 2: Like the previous one, but the third point of each vehicle's path will be changed.

Onlooker 3: The previous method is performed for two points. So two points of each vehicle's path will be given to the next vehicle.

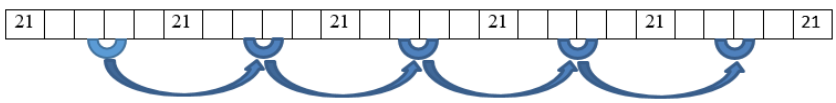

Figure 3. The format of Onlooker 3 
Onlooker 4: The previous method will be done for two other points.

4.2.2 Close Neighbourhood: In this method, the answers are improved by making small changes.

4 Onlooker are produced as follows:

Onlooker 1: one point of the first 8 points of the string is changed with 1 random point from the remaining 8th point.

Onlooker 2: It is like the first method, but it has done between the two sides of the path, so that one point between 1st and 13th changes with one point from 13th to 26th.

Onlooker 3: Like the previous one, the exchange between the first 18 points and the next 8 points is applied.

Onlooker 4: The previous method is performed at two points randomly.

\subsection{Improving the process using Genetic Algorithm}

A genetic algorithm is a search heuristic that is inspired by Charles Darwin's theory of natural evolution. This algorithm reflects the process of natural selection where the fittest individuals are selected for reproduction in order to produce offspring of the next generation.

Genetic Algorithm is used in the Onlooker bee production stage. After generating a neighbouring solution, the genetic algorithm is implemented separately for each vehicle and the path of each vehicle is optimized.

\subsection{Fitness Function}

In this problem, the sum of the travelled routes by five devices is considered as the fitness function and is intended to minimize this function throughout the program. Note that the distance between points is based on Euclidean distance.

\subsection{Implementation}

At the first step, 50 scout bees are produced. Then, according to the fitness function, five of the above-mentioned populations are selected and proceed to the next step as the best answers. Then for each of these bees, 4 onlooker bees are produced, so 25 scout bees are produced to generate a new 50 bees. A repetitive production of a new population from current population is carried out 1000 times. The best answer in the final step is considered as the final answer to our problem.

According to the methods mentioned above and the combination of these methods, eight scenarios are implemented as follows:

1. The production of scout bee is based on nearest neighbourhood method and the production of onlooker bee is done by wide neighbourhood method.

2. The production of scout bee is based on nearest neighbourhood method and the production of onlooker bee is done by wide neighbourhood method and GA.

3. The production of scout bee is based on nearest neighbourhood method and the production of onlooker bee is done by nearest neighbourhood method.

4. The production of scout bee is based on nearest neighbourhood method and the production of onlooker bee is done by nearest neighbourhood method and GA.

5 . The production of scout bee is based on random method and the production of onlooker bee is done by wide neighbourhood method.
6. The production of scout bee is based on random method and the production of onlooker bee is done by wide neighbourhood method and GA.

7. The production of scout bee is based on random method and the production of onlooker bee is done by nearest neighbourhood method.

8. The production of scout bee is based on random method and the production of onlooker bee is done by nearest neighbourhood method and GA.

In the next part, implemented scenarios are shown as maps. Central point in each map is the start point that all five vehicles start their routes. Each vehicle has a different colour to identify. The breaks in vehicle's routes represents travellers. Every vehicle starts from the central point, pickup four travellers and returns to central point and the length of its route will be calculated. All five vehicle's routes will add up and the best answer is the shortest one. Results will be shown in part 5 .

\section{RESULTS}

All scenarios run 10 times and two parameters are measured: a) Average value of answer (total length of five vehicles) in meters, b) Average time of program implementation in seconds. Results will be shown in Fig. 4 to Fig. 11.

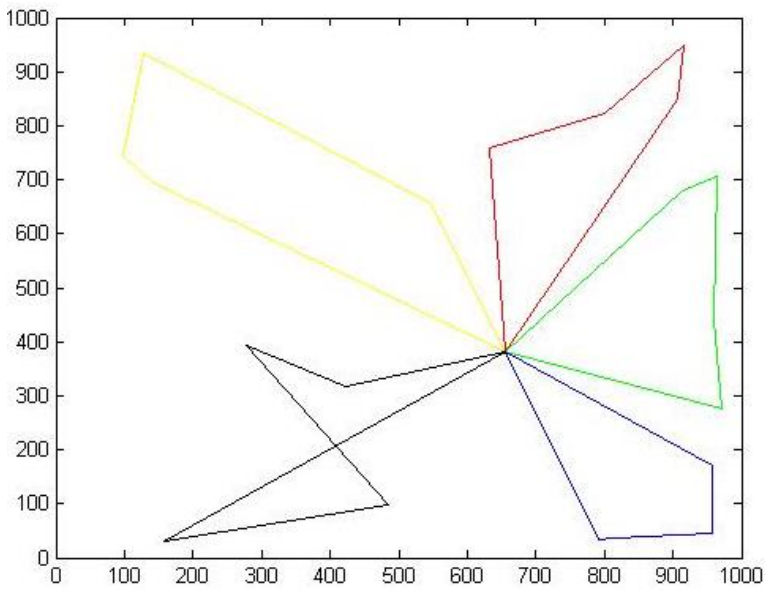

Figure 4. Scenario I, Average answer is $6971.51 \mathrm{~m}$, average time is $45 \mathrm{~s}$.

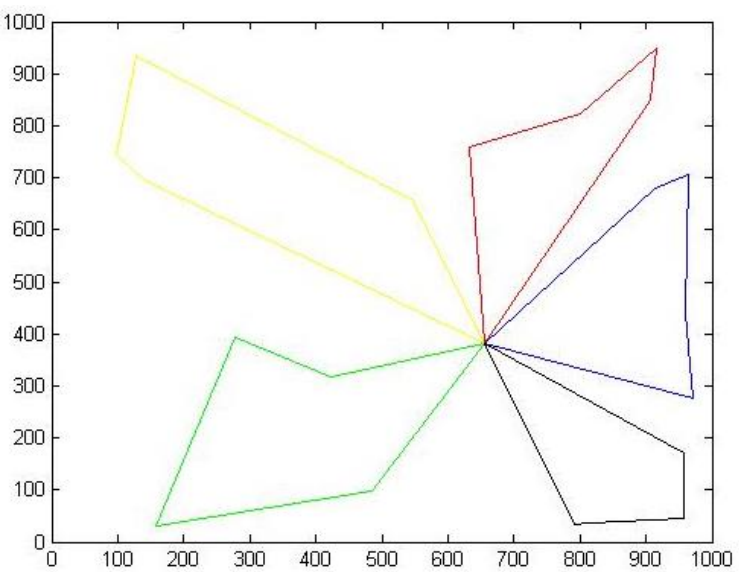

Figure 5. Scenario II, Average answer is $6765.63 \mathrm{~m}$, average time is $27.57 \mathrm{~s}$. 


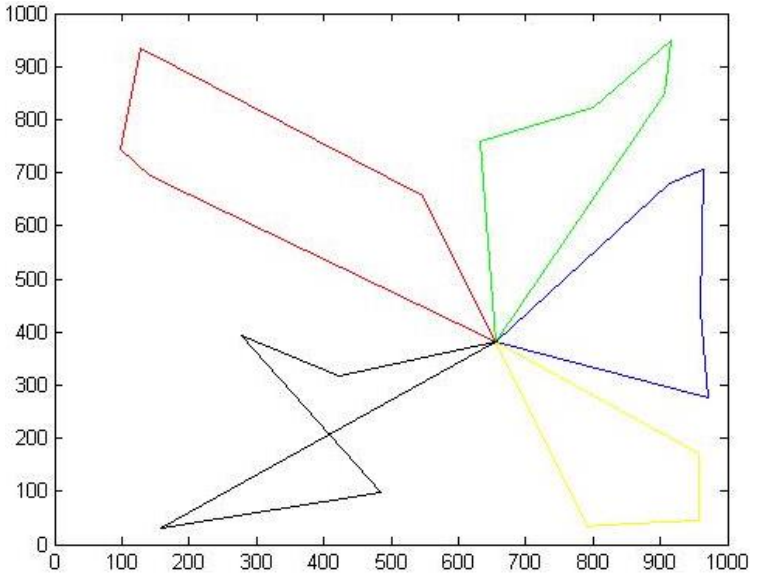

Figure 6. Scenario III, Average answer is $6971.15 \mathrm{~m}$, average time is $60.23 \mathrm{~s}$.

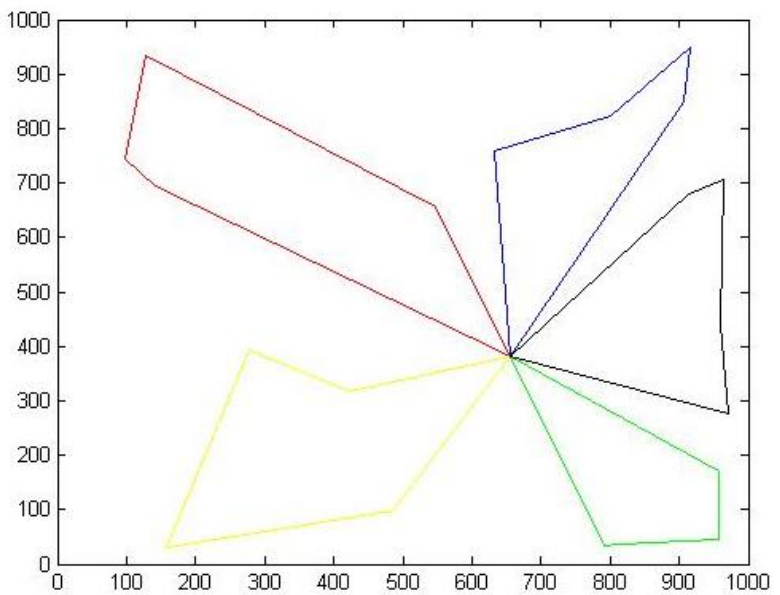

Figure 7. Scenario IV, Average answer is $6714.16 \mathrm{~m}$, average time is $279.25 \mathrm{~s}$

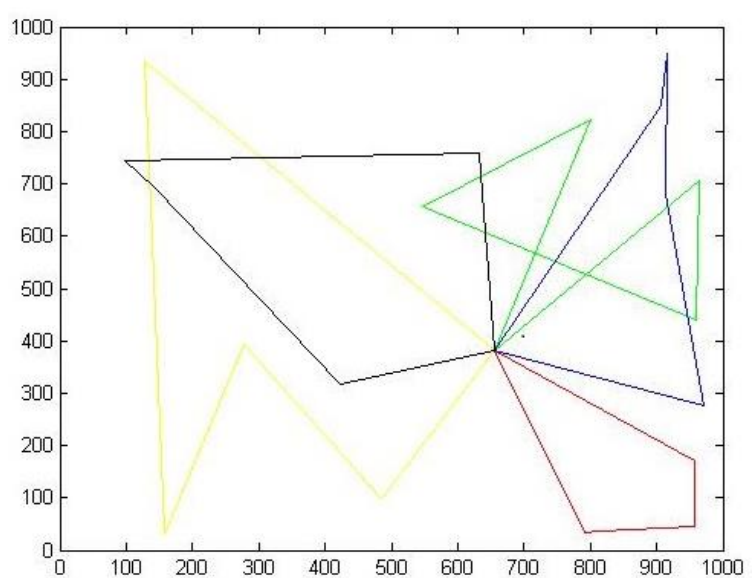

Figure 8. Scenario V, Average answer is $9735.87 \mathrm{~m}$, average time is $4.08 \mathrm{~s}$.

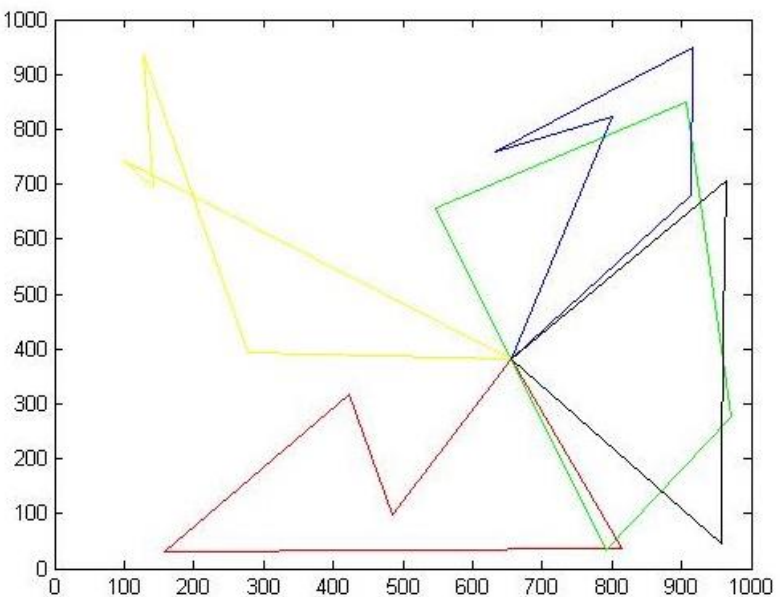

Figure 9. Scenario VI, Average answer is $8608.41 \mathrm{~m}$, average time is $195.9 \mathrm{~s}$.

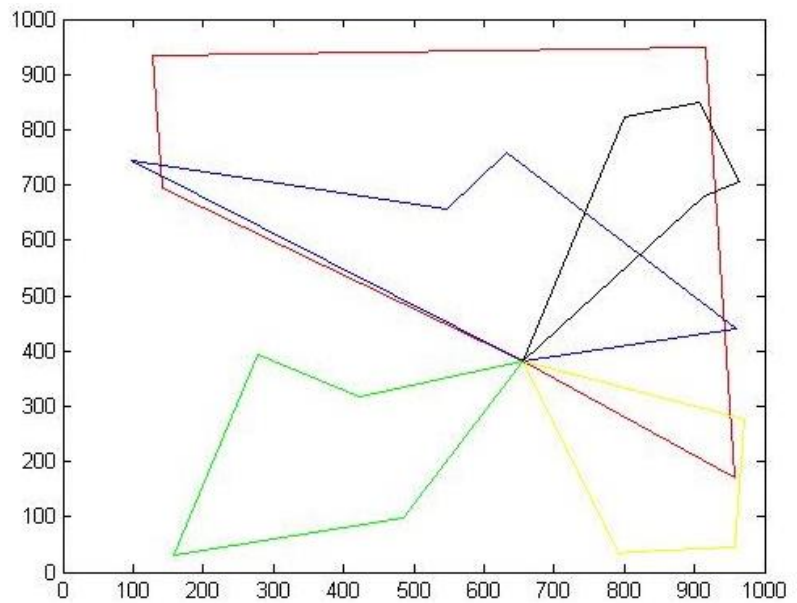

Figure 10. Scenario VII, Average answer is $9244.15 \mathrm{~m}$, average time is $4.07 \mathrm{~s}$.

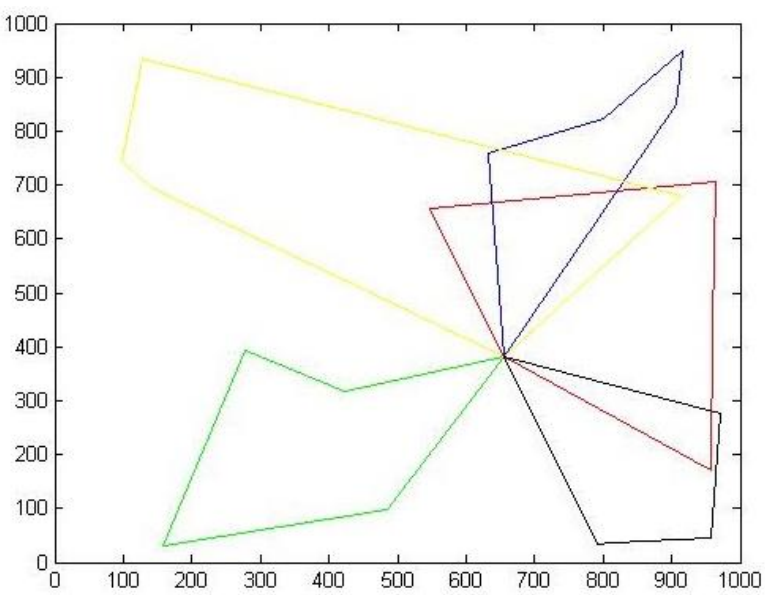

Figure 11. Scenario VIII, Average answer is $8657.8 \mathrm{~m}$, average time is $615.28 \mathrm{~s}$ 


\section{CONCLUSION}

All average answers and average times is shown in Figure. 12 and Figure. 13.

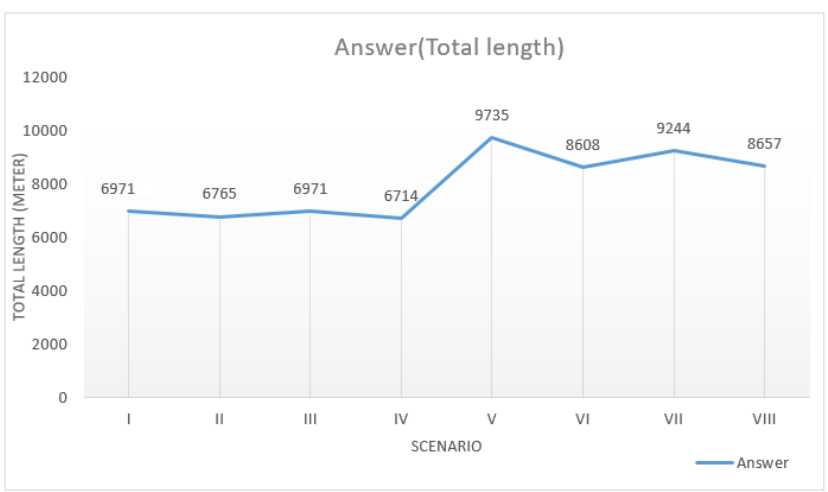

Figure 12. Average answers (Total length)

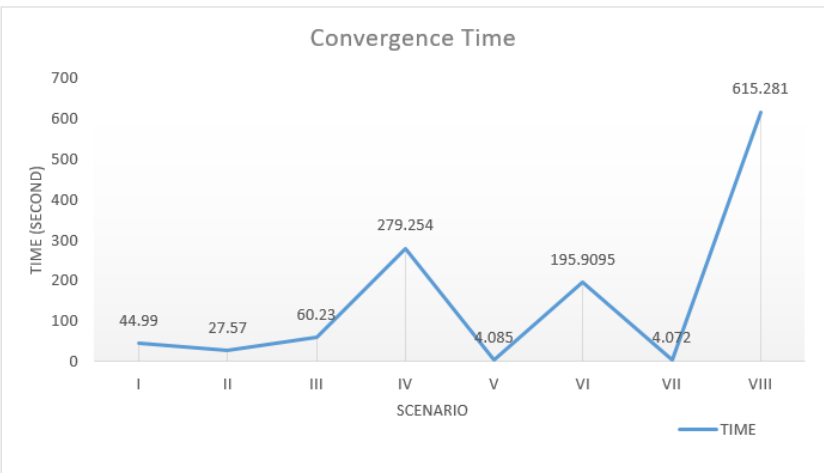

Figure 13. Average times

Since this problem is kind of NP-Hard problems we have to use metaheuristic methods and it cannot be solved by simple algorithms such as simple Dijkstra. Thus, there is no baseline approach to compare the results; in fact, in this paper, the focus is on metaheuristic algorithms and the comparison between their performances.

As shown in the results section, the most sustainable and most reliable answer is scenario IV, which is longer than other scenarios in terms of runtime. Since the problem just runs once, and does not run online like route finding, so being time consuming is not a weak point for this algorithm. However, the sustainability and optimality of the answer is the most crucial issue.

The comparison of the results shows that the production of scout bees is the most effective factor in the answer of the program, also it helps greatly converging the answer as soon as possible and optimizes it.

When scout bees are used in the nearest neighborhood method, the used method for the onlooker bees does not differ significantly, but using the genetic algorithm causes a huge difference in the answers.

If random method is used to produce scout bee, a large difference in the final answer of the problem will be caused by the inappropriateness of this method. And if this method is used, although the program time is reduced, but the optimal answer will be never reached.

Although there are only 5 vehicles (and 20 customers) and the running time is approximately 280 seconds, it is a theoretical experiment; certainly, if we want to implement it in a great scale and use it as a real service (mobile or web application), we will use advanced software and hardware to decrease the running time and use it dynamically and serve thousands of clients.

The wide or nearest neighborhood method and genetic algorithm does not converge the final answer to the optimal answer. However, using nearest neighborhood and genetic algorithm is the best way to produce the onlooker bee.

\section{REFERENCES}

Brandão, J., 2018. Metaheuristic for the Vehicle Routing Problem with Backhauls and Time Windows. Operations Research Proceedings 2017, 413-418.

Gökçe, E.İ., 2004. A revised ant colony system approach to vehicle routing problems. Masther's Thesis, Graduate School of Engineering and Natural Sciences, Sabanci University, Turkey.

Karaboga, D., 2005. An idea based on honey bee swarm for numerical optimization (Vol. 200). Technical report-tr06, Erciyes university, engineering faculty, computer engineering department.

Kumar, S., Sharma, V.K., Kumari, R., 2014. Improved onlooker bee phase in artificial bee colony algorithm. arXiv preprint arXiv: 1407.5753 .

Marinakis, Y., Marinaki, M., Migdalas, A., 2017. Particle swarm optimization for the vehicle routing problem: a survey and a comparative analysis. Handbook of Heuristics, 1-34.

Marinakis, Y., Marinaki, M., Migdalas, A., 2019. A MultiAdaptive Particle Swarm Optimization for the Vehicle Routing Problem with Time Windows. Information Sciences, 481, 311329.

Najibi, N., Arabsheibani, R., 2013. Snow-covered surface variability and DEM generation using aerial photogrammetry in Mount Odin, Canada. Geodesy and Cartography, 39(3), 113120.

Pham, D.T., Ghanbarzadeh, A., Koç, E., Otri, S., Rahim, S., Zaidi, M., 2006. The bees algorithm-a novel tool for complex optimisation problems. In Intelligent Production Machines and Systems, 454-459. Elsevier Science Ltd.

Purves, W.K., Sadava, D., Orians, G.H., Heller, H.C., 2003. Life: the science of biology: volume III: plants and animals. Macmillan.

Seeley, T.D., Visscher, P.K., 1988. Assessing the benefits of cooperation in honeybee foraging: search costs, forage quality, and competitive ability. Behavioral Ecology and Sociobiology, 22(4),229-237.

Tsai, P.W., Yang, L.H., Zhang, J., Zhang, Y.H., Chang, J.F., Istanda, V., 2016, October. Composing High Event Impact Resistible Model by Interactive Artificial Bee Colony for the Foreign Exchange Rate Forecasting. In International Conference on Advanced Intelligent Systems and Informatics, 760-770. Springer, Cham. 